\title{
BMJ Open External barriers for including parents of preterm infants in a randomised clinical trial in the neonatal intensive care unit in Sweden: a descriptive study
}

\author{
Evalotte Mörelius (D) , 1,2 Emma Olsson (D) , 3,4 Charlotte Sahlén Helmer, ${ }^{1}$ \\ Ylva Thernström Blomqvist, ${ }^{5,6}$ Charlotte Angelhoff (iD ${ }^{7}$
}

To cite: Mörelius E, Olsson E, Sahlén Helmer C, et al. External barriers for including parents of preterm infants in a randomised clinical trial in the neonatal intensive care unit in Sweden: a descriptive study. BMJ Open 2020;10:e040991. doi:10.1136/ bmjopen-2020-040991

- Prepublication history for this paper is available online. To view these files, please visit the journal online (http://dx.doi org/10.1136/bmjopen-2020040991).

Received 28 May 2020 Revised 12 November 2020 Accepted 22 November 2020

Check for updates

(C) Author(s) (or their employer(s)) 2020. Re-use permitted under CC BY-NC. No commercial re-use. See rights and permissions. Published by BMJ.

For numbered affiliations see end of article.

Correspondence to Professor Evalotte Mörelius; evalotte.morelius@liu.se

\section{ABSTRACT}

Objectives Performing randomised controlled trials (RCTs) in neonatal intensive care is challenging in many ways. While restrictive inclusion criteria or busy study protocols are obvious barriers, external barriers leading to termination of a study are seldom discussed. The aim of this study was to describe barriers for inclusion of families in neonatal intensive care in an RCT aiming to evaluate the effects of continuous skin-to-skin contact on mood and sleep quality in parents of preterm infants, as well as the quality of parent-infant interaction and salivary cortisol concentrations at the time of discharge.

Design A descriptive study.

Setting Three out of seven tertiary neonatal intensive care units in Sweden participated in a two-arm RCT that was terminated because of low inclusion rate.

Participants Before termination of the study, 11 out of 242 families assessed for eligibility were included for participation.

Results The major barriers for inclusion in this RCT were external due to (1) lack of intensive care beds in the neonatal ward, causing medically stable infants to be transferred back to the referring hospital quicker than expected, (2) moving directly from the delivery room to a family room without passing an open bay intensive care room or (3) transferring from one neonatal ward to another with the same care level to increase availability of intensive care beds where needed. Other barriers were the inclusion criteria 'single-birth' and 'Swedish-speaking parent'.

Conclusions The major barriers for including participants were external constituted by transferals between neonatal wards and cities due to lack of intensive care beds. This is a multifactorial issue related to organisational structures. However, since this affects the possibilities to perform research this study highlights some suggestions to consider when planning prospective intervention studies within a neonatal setting.

Trial registration number NCT03004677.

\section{INTRODUCTION}

Around the world, 15 million infants are born preterm every year. ${ }^{1}$ In Sweden, approximately $6 \%$ of the infants are born before gestational week $38 .^{2}$ Thanks to advances in medical
Strengths and limitations of this study

- Few studies discuss early termination of studies in neonatal intensive care.

- This study describes external barriers for including families in a randomised controlled study.

- A negative outcome related to the termination of this study is that we still do not know if continuous skin-to-skin contact starting after the intensive care period would function as a restart for bonding and therefore improve the parents' mood, interaction behaviour and coregulation of salivary cortisol between parent and infant.

treatment, there is an increased survival rate for infants born extremely preterm. ${ }^{3}$ However, these infants require extensive neonatal intensive care for several weeks and hospital care for several months. ${ }^{3}$ Separation between parent and infant is common since many parents do not have the opportunity to stay around the clock in the neonatal intensive care unit (NICU). ${ }^{4}$ A separation of the parent-infant dyad is more common during the intensive care period than after, when more hospitals can offer couplet care and single family rooms. ${ }^{5}$ Separation in combination with the infants' health condition and need of intensive care exposes parents to distress, worries and poor sleep. This increases the risk for allostatic overload, postpartum depression and post-traumatic stress disorder ${ }^{467}$ which in turn increases the risk for poor bonding and parent-infant interaction. ${ }^{89}$

Practising skin-to-skin contact (SSC) during the NICU stay is one way to minimise separation and instead increase physical contact and possibilities for interaction between the parent and the infant. ${ }^{10}$ In SSC, parents carry the infant in an upright position skin to skin on their chest, which improves parents' mood and reduce stress and symptoms of postpartum depression. ${ }^{11} 12$ 
Neonatal research often aims to find new interventions to improve care for preterm infants and their families. However, conducting a randomised controlled trial (RCT) in the NICU is challenging in many ways, including recruiting participants. According to Williams et al, the most common reason for terminating clinical trials is insufficient accrual rate. ${ }^{13} \mathrm{~A}$ review by McDonald et al showed that only $31 \%$ of the 114 included trials met their original recruitment goal. ${ }^{14}$ In $34 \%$ of the trials, the sample size was reduced during the trial, in $11 \%$ recruitment was terminated before the formal end of enrolment and in $10 \%$ the inclusion criteria were changed as a way of improving recruitment. ${ }^{14}$ To better understand the barriers impeding recruitment for patients with cancer, Stafford et al conducted interviews with recruiters. ${ }^{15}$ Some of the barriers they found for insufficient accrual rate were delays in receiving multisite ethics and governance approval, physical relocation of one of the recruitment sites, timing of the study in relation to the participant's cancer journey and perceived burden of participation. ${ }^{15}$ While an obvious problem can be that researchers specify inclusion criteria that are too restrictive or aim to include more tasks than the participant can handle, external circumstances out of the researchers' power to influence, are seldom discussed.

In 2018, we published a study protocol for an RCT with the aim to evaluate the effect of continuous SSC on sleep quality and mood in parents of preterm infants as well as the quality of parent-infant interaction and salivary cortisol concentrations at the time of discharge. ${ }^{16}$ The hypothesis was that continuous SSC starting after the intensive care period would function as a restart for bonding and therefore improve the parents' mood, interaction behaviour and coregulation of salivary cortisol between parent and infant. Furthermore, it was hypothesised that the parents' possibility to take turns and assist each other with practical support during SSC would facilitate parents' sleep quality. The study started and opened for recruitment in January 2017 but due to low inclusion rate, the study was finally terminated in March 2020.

\section{AIM}

The purpose of this paper was to describe barriers for inclusion of families in neonatal intensive care in an RCT aiming to evaluate the effects of continuous SSC on sleep quality and mood in parents of preterm infants, as well as the quality of parent-infant interaction and salivary cortisol concentrations at the time of discharge.

\section{METHODS}

\section{Description of the terminated study}

The protocol for the terminated two-arm (intervention and control) RCT is published. ${ }^{16}$

\section{Participants}

Three out of seven tertiary university hospitals in Sweden providing care for extremely preterm infants were included. The settings were family-centred NICUs with single family rooms where the family can stay together with their infant around the clock when extensive intensive care is no longer required. The parents' presence at NICUs in Sweden are facilitated through the Swedish national insurance system, which allows both parents to share parental benefit for 480 days per child. In addition, both parents of an infant requiring care at an NICU are entitled to temporary parental benefit until the infant is discharged. This means that during the infant's entire NICU stay, both parents have the legal right to be together with their infant at the NICU. Moreover, hospital care in Sweden is free of charge for all children.

Inclusion criteria were parents (mother and father/ partner) of single preterm infants born $<30$ weeks of gestation. The infant should have moved out from the open bay intensive care room and into a single family room where both parents could stay and care for their infant all hours of the day. Parents should be able to read and understand Swedish. Exclusion criteria were parents with sleeping disorders, psychiatric problems or drug abuse and infants with major congenital malformation, grade III-IV intraventricular haemorrhage or a chromosomal defect that could affect the infant's ability to interact. In agreement with a power calculation, the aim was to include 50 parents ( $=25$ families). Based on previous numbers of admittance, it was estimated to take 1 year to recruit these families from the three different sites.

\section{Procedure}

Research coordinators on each unit identified potential families and provided the parents with written and oral information about the study prior to moving to a family room. The families who accepted to participate were randomised to the intervention or control group before they moved into the family-room. In families assigned to the intervention, the infants should be cared for in continuous SSC on parents' chests, 24hours a day, for four consecutive days, alternating between the parents. Families randomised to the control group were free to continue to practise intermittent SSC as much as they wanted since this was part of the standard care in the participating NICUs. ${ }^{17}$ Outcome measurements included: parents' mood, sleep (actigraphy) and sleep quality, parent-infant interaction, and salivary cortisol reactivity, recovery and coregulation (for further information please see the study protocol). ${ }^{16}$

\section{Patient and public involvement}

The protocol was pilot tested with two families before the study commenced. The families provided feedback on the study sequence and feasibility. ${ }^{16}$ 


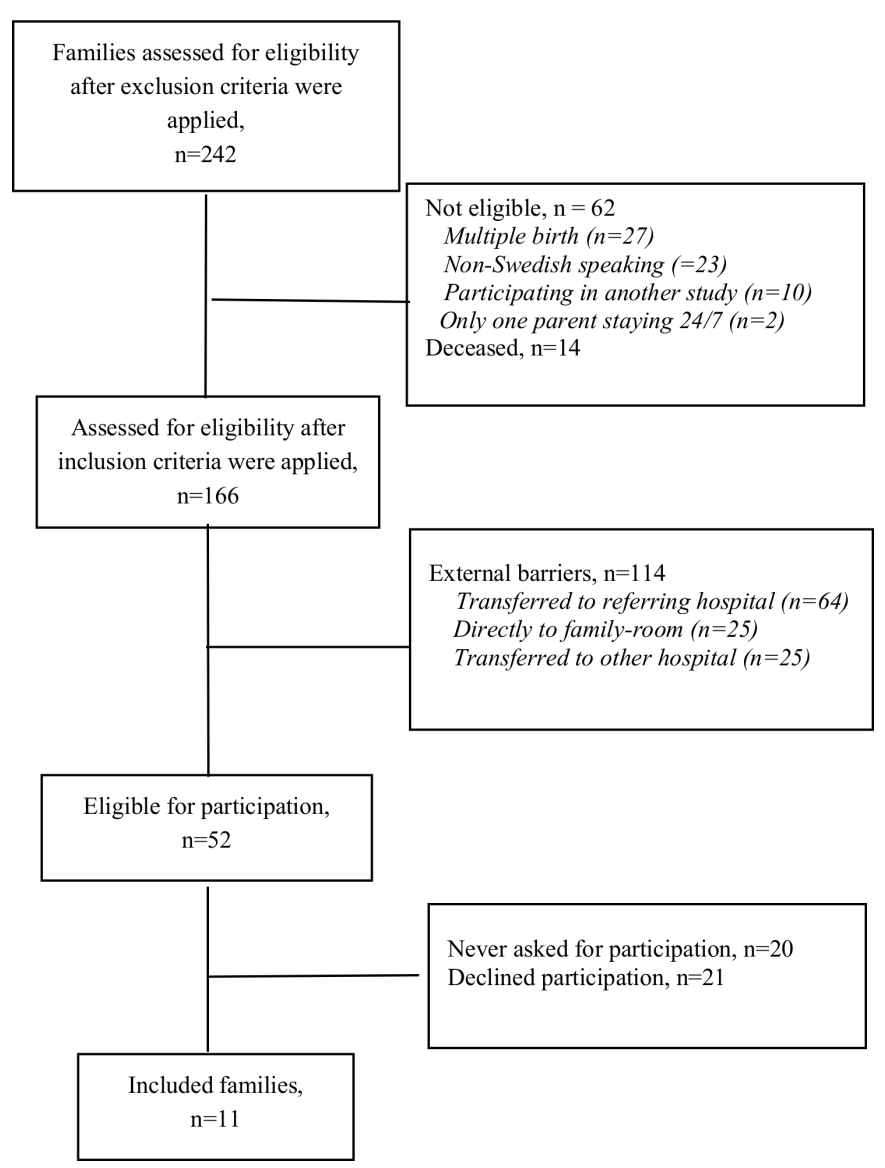

Figure 1 Flow chart of eligibility, barriers for inclusion and included families.

\section{RESULTS}

A total of 242 families were assessed for eligibility whereof 11 were included (figure 1). The major barriers for inclusion in this study were external barriers $(n=114)$. External barriers were related to (1) the lack of neonatal intensive care beds in Sweden at the time, causing medically stable infants to be transferred back to the referring hospital quicker than usual ( $\mathrm{n}=64)$, (2) moving directly from the delivery room to a family room without the need for intensive care $(n=25)$ or (3) moving from hospital to hospital across the country to increase availability of neonatal intensive care beds where needed $(n=25)$.

Another identified barrier for enrolment was the inclusion criteria $(n=62)$. Multiple birth was not included in this study because of the higher demands of practising continuous SSC with more than one infant $(n=27)$. NonSwedish-speaking parents were not included due to lack of validated translations of the included questionnaires $(\mathrm{n}=23)$.

Some families were never asked for participation despite eligibility $(n=20)$. The reasons were that the research coordinators did not manage to approach the families in time because of a high patient volume, lack of staff, or a short admission time at the included NICU before moving to another hospital.

Twenty-one parents declined participation, reasons for declining were not recorded. Some efforts were made to adjust the protocol in order to facilitate recruitment. Gestational age of inclusion criteria was changed from $<30$ weeks to $<33$ weeks to increase the number of potential candidates. The time in continuous SSC was decreased from four consecutive nights to 24 hours because some parents had expressed that four nights was a long time period for them to manage the intervention, or that they did not have the energy to comply to a study for several days. Moreover, in an attempt to ease the burden of participation, the documentation of sleep and time in SSC were simplified and the actigraph device was set on the ankle instead as some parents had complained over itching and difficulties to sleep when they wore the device around the waist. Despite these changes, there were no noticeable change in recruitment. In total, 11 families where included in the study before termination because of insufficient inclusion rate (figure 1).

\section{DISCUSSION}

The overall reason for terminating this study was due to the external barrier of a lack of intensive care beds in the participating hospitals. In some countries, infants and their families may be transferred from one NICU to another during their hospitalisation either because of a lack of available beds or the need for another level of care. Transferal of infants who no longer require intensive care, from tertial to non-tertial NICUs is routine in Sweden today because extremely preterm infants are at less risk of mortality and morbidity if born in a tertiary hospital. ${ }^{18}$ In times of high patient volume and lack of staff, there may be a need to identify which infants have least need for intensive care thus transferal from an intensive care room to a family room within the same NICU may be an option. Transferal between NICUs with the same level of care is more difficult to explain and often unpredictable for families as well as for researchers, increasing the risk for parental stress and problems with recruitment. Multiple transferals may lead to families receiving neonatal care in several NICUs across the country, making it difficult to comply to clinical intervention protocols especially if the intervention is a prospective single site study. For this purpose, it is important that non-tertiary NICUs also participate and fulfil clinical intervention protocols initiated at tertiary hospitals, which warrants more clinical researchers in these hospitals. However, transferals between same level NICUs are more an organisation issue partially due to lack of staff to meet a high patient volume.

Risks and benefits with transferral from a tertiary to a non-tertial hospital has been discussed for years ${ }^{19}$ and a meta-analysis comprising 12 articles of parents' experiences show that neonatal transfer can be scary and threating but also a relief to be closer to home. ${ }^{20}$ Less is known about parents' experiences of transferals between different NICUs with same level of care due to a lack of intensive care beds.

Having twins and language barriers were the most common reason for ineligibility for the terminated study. 
Twins have a higher risk of being born preterm and needing neonatal care and constitutes a large proportion of NICU admissions. ${ }^{21}$ However, to include families of twins in the terminated study was not feasible since the parents were supposed to assist and support each other by taking turns providing continuous SSC. Non-Swedishspeaking parents is a growing group and the use of translators and interpreters need to be considered in the future in order to be inclusive and increase generalisability. ${ }^{22}$

DeMauro et al suggest that strategies to reduce the burden of research participation in the NICU may facilitate participation. Furthermore, that low maternal education, larger families, site and public insurance affect attrition rate. ${ }^{23}$ Due to ethical restrictions, we have no data on families who declined participation and can therefore only relate to the factor of public insurance. As public insurance applies to all families in Sweden it is not suggested to be a factor that explains attrition rate in the present study. Lack of demographic data also limits the possibility to compare parents who declined with parents who agreed to participate. However, the major problem with the terminated study was the inclusion rate. Even though a pilot test for feasibility was performed before the start of the study and efforts were made to ease the burden of participation, the inclusion rate remained low.

When and how to approach parents for consent can impact their decision. According to Korotchikova et al, a significantly higher number consent to participation in research if both parents are present when approached. ${ }^{24}$ In the terminated study, parents were approached in connection to the move from the intensive care room to the single family room which might have provided some stress for the parents with a lot of changes happening at the same time. However, 114 families were never approached due to external barriers.

Being a parent to a premature infant in the NICU is an emotional experience extending for a long period of time. Couplet care and family-centred care is a relatively new phenomenon in neonatal intensive care after a history of separation between parents and preterm infants during admission to an NICU. This is appreciated by the parents but is also a demanding task and sometimes a stressful experience. ${ }^{12}{ }^{25}$ We did not ask the 21 families who declined participation about the reasons. It is possible that the stressful situation was a contributing factor since one third of parents refusing consent in another study of neonatal research did so because of tiredness and stress. ${ }^{24}$ One objective with the terminated study was to evaluate if sleep quality was facilitated when the parents took turns and assisted each other with practical support during SSC. This is a dilemma where parents are potentially too exhausted to engage in research that potentially could be beneficial for their well-being. Parents' previous experiences of intermittent SSC could also have influenced the parents' choice in participating in the study. We did not collect data on parents' previous experiences or to what extent they had practised intermittent SSC since this is part of the standard care. Sweden has a long tradition of practising intermittent $\mathrm{SSC}^{26}$ and most of the extremely preterm infants experience their first intermittent SSC session within the first week of life. ${ }^{17}$

The issues raised in this paper are important to consider when planning an experimental study in an NICU in Sweden and other countries that share similar characterisation of the public health system. In different hospital structures, there might be other internal or external factors hindering consent. ${ }^{1415}$ However, studies within an NICU context are few and focus on withdrawal and attrition rather than barriers for inclusion, ${ }^{23}{ }^{27}$ which warrant more research within this field.

\section{CONCLUSIONS}

The major reason why some participants were not included was due to the external barrier constituted by transferals between NICUs. This is a multifactorial issue related to organisational structures. Still, an important issue for researchers to consider when planning prospective intervention studies within an NICU setting. Drawn from the results of this study, our advises for future trials comprise of: performing multicentred studies including several tertiary NICUs, including researchers from non-tertiary hospitals, considering if it is possible to include families with multiple birth, increasing the use of interpreters in order to be inclusive of all families, and including parents in the study design. A negative outcome related to the termination of this study is that we still do not know if continuous SSC starting after the intensive care period would function as a restart for bonding and therefore improve the parents' mood, sleep quality, interaction behaviour and coregulation of salivary cortisol between parent and infant.

\section{Author affiliations}

${ }^{1}$ Department of Health, Medicine and Caring Sciences, Linköping University, Linköping, Sweden

${ }^{2}$ School of Nursing and Midwifery, Edith Cowan University, Joondalup, Western Australia, Australia

${ }^{3}$ School of Health Sciences, Örebro University, Örebro, Sweden

${ }^{4}$ Department of Pediatrics, University Hospital Örebro, Örebro, Sweden

${ }^{5}$ Department of Women's and Children's Health, Uppsala University, Uppsala, Sweden

${ }^{6}$ Neonatal Intensive Care Unit, Uppsala University Hospital, Uppsala, Sweden ${ }^{7}$ Crown Princess Victoria's Child and Youth Hospital and Department of Biomedical and Clinical Sciences, Linköping University, Linköping, Sweden

Twitter Evalotte Mörelius Twitter@EvalotteM and Charlotte Angelhoff @Angelhoff_C

Acknowledgements The authors would like to thank Dr Stephanie Smith, Edith Cowan University, School of Nursing and Midwifery, Joondalup, Western Australia for help to revise the language.

Contributors All authors contributed to the study design. EM had primary responsibility of writing the paper. CA, EO, CSH and YTB critically reviewed and approved the manuscript.

Funding This work was supported by Medical Research Council of Southeast Sweden (FORSS -661721), Region Östergötland, Sjukdom och Välfärd, (LIO720151) and Region Östergötland, Forsknings-och Stipendieförvaltningen (LIO-663781).

Competing interests None declared. 
Patient and public involvement Patients and/or the public were not involved in the design, or conduct, or reporting, or dissemination plans of this research.

Patient consent for publication Not required.

Ethics approval The study was approved by the Regional Ethics Committee, Linköping University (2016/89-31, 2017/566-32).

Provenance and peer review Not commissioned; externally peer reviewed.

Data availability statement Data sharing not applicable as no datasets generated and/or analysed for this study. Data used for the study are available from the corresponding author on request.

Open access This is an open access article distributed in accordance with the Creative Commons Attribution Non Commercial (CC BY-NC 4.0) license, which permits others to distribute, remix, adapt, build upon this work non-commercially, and license their derivative works on different terms, provided the original work is properly cited, appropriate credit is given, any changes made indicated, and the use is non-commercial. See: http://creativecommons.org/licenses/by-nc/4.0/.

\section{ORCID iDs}

Evalotte Mörelius http://orcid.org/0000-0002-3256-5407

Emma Olsson http://orcid.org/0000-0002-5582-6147

Charlotte Angelhoff http://orcid.org/0000-0002-0174-8630

\section{REFERENCES}

1 WHO. Preterm birth, 2018. Available: http://www.who.int/en/news$\mathrm{room} /$ fact-sheets/detail/preterm-birth [Accessed 1 Nov 2020].

2 Swedish neonatal quality register, SNQR, 2018. Available: https:// www.medscinet.com/PNQ/uploads/website/arsrapporter/ Neonatalregistrets\%20Årsrapport\%202018._3.0.pdf2020

3 Norman M, Hallberg B, Abrahamsson T, et al. Association between year of birth and 1-year survival among extremely preterm infants in Sweden during 2004-2007 and 2014-2016. JAMA 2019;321:1188-99.

4 Flacking R, Lehtonen L, Thomson G, et al. Closeness and separation in neonatal intensive care. Acta Paediatr 2012;101:1032-7.

5 Tandberg BS, Frøslie KF, Flacking R, et al. Parent-Infant Closeness, parents' participation, and nursing support in Single-Family room and open Bay NICUs. J Perinat Neonatal Nurs 2018;32:E22-32.

6 Schecter R, Pham T, Hua A, et al. Prevalence and longevity of PTSD symptoms among parents of NICU infants analyzed across gestational age categories. Clin Pediatr 2020;59:163-9.

7 Loewenstein K, Barroso J, Phillips S. The experiences of parents in the neonatal intensive care unit: an integrative review of qualitative studies within the Transactional model of stress and coping. J Perinat Neonatal Nurs 2019;33:340-9.

8 Dubber S, Reck C, Müller M, et al. Postpartum bonding: the role of perinatal depression, anxiety and maternal-fetal bonding during pregnancy. Arch Womens Ment Health 2015;18:187-95.

9 Kerstis B, Aarts C, Tillman C, et al. Association between parental depressive symptoms and impaired bonding with the infant. Arch Womens Ment Health 2016;19:87-94.

10 Mörelius E, Örtenstrand A, Theodorsson E, et al. A randomised trial of continuous skin-to-skin contact after preterm birth and the effects on salivary cortisol, parental stress, depression, and breastfeeding. Early Hum Dev 2015;91:63-70.

11 Scime NV, Gavarkovs AG, Chaput KH. The effect of skin-to-skin care on postpartum depression among mothers of preterm or low birthweight infants: a systematic review and meta-analysis. J Affect Disord 2019;253:376-84.

12 Edéll-Gustafsson U, Angelhoff C, Johnsson E, et al. Hindering and buffering factors for parental sleep in neonatal care. A phenomenographic study. J Clin Nurs 2015;24:717-27.

13 Williams RJ, Tse T, DiPiazza K, et al. Terminated trials in the ClinicalTrials.gov results database: evaluation of availability of primary outcome data and reasons for termination. PLoS One 2015;10:e0127242.

14 McDonald AM, Knight RC, Campbell MK, et al. What influences recruitment to randomised controlled trials? A review of trials funded by two UK funding agencies. Trials 2006;7:9.

15 Stafford L, Sinclair M, Newman L, et al. Why did we fail? Challenges recruiting parents with cancer into a psycho-educational support program. Psychooncology 2019;28:2425-8.

16 Angelhoff C, Blomqvist YT, Sahlén Helmer C, et al. Effect of skin-toskin contact on parents' sleep quality, mood, parent-infant interaction and cortisol concentrations in neonatal care units: study protocol of a randomised controlled trial. BMJ Open 2018:8:e021606.

17 Mörelius E, Angelhoff C, Eriksson J, et al. Time of initiation of skin-toskin contact in extremely preterm infants in Sweden. Acta Paediatr 2012;101:14-18.

18 Helenius K, Longford N, Lehtonen L, et al. Association of early postnatal transfer and birth outside a tertiary hospital with mortality and severe brain injury in extremely preterm infants: observational cohort study with propensity score matching. BMJ 2019;367:15678.

19 Donohue PK, Hussey-Gardner B, Sulpar LJ, et al. Convalescent care of infants in the neonatal intensive care unit in community hospitals: risk or benefit? Pediatrics 2009;124:105-11.

20 Aagaard H, Hall EOC, Ludvigsen MS, et al. Parents' experiences of neonatal transfer. A meta-study of qualitative research 2000-2017. Nurs Inq 2018;25:e12231.

21 Martin JA, Hamilton BE, Osterman MJK, et al. Births: final data for 2015. Natl Vital Stat Rep 2017;66:1.

22 Statistics Sweden. Most immigrants were Swedish-born persons 2019, 2020. Available: https://www.scb.se/en/finding-statistics/ statistics-by-subject-area/population/population-composition/ population-statistics/pong/statistical-news/population-statisticsjanuaryjune-2019/ [Accessed 27 May 2020].

23 DeMauro SB, Bellamy SL, Fernando M, et al. Patient, family, and center-based factors associated with attrition in neonatal clinical research: a prospective study. Neonatology 2019;115:328-34.

24 Korotchikova I, Boylan GB, Dempsey EM, et al. Presence of both parents during consent process in non-therapeutic neonatal research increases positive response. Acta Paediatr 2010;99:1484-8.

25 Ndiaye S, Bosowski J, Tuyisenge L, et al. Parents as carers on a neonatal unit: qualitative study of parental and staff perceptions in a low-income setting. Early Hum Dev 2020;145:105038.

26 Wahlberg V. Alternative care for premature infants the kangaroo method. Advantages, risks and ethical questions. Neonatologica 1987;4:363-7.

27 Guillén U, DeMauro S, Ma L, et al. Relationship between attrition and neurodevelopmental impairment rates in extremely preterm infants at 18 to 24 months: a systematic review. Arch Pediatr Adolesc Med 2012;166:178-84. 